\title{
Efficacy of Retinal Spikes in Driving Cortical Responses
}

\author{
Prakash Kara and R. Clay Reid \\ Department of Neurobiology, Harvard Medical School, Boston, Massachusetts 02115
}

\begin{abstract}
How does a single retinal ganglion cell (RGC) affect the firing of simple cells in the visual cortex? Although much is known of the functional connections between the retina and the lateral geniculate nucleus (LGN) and between LGN and visual cortex, it is hard to infer the effect of disynaptic connections from retina to visual cortex. Most importantly, there is considerable divergence from retina to LGN, so cortical neurons might be influenced by ganglion cells through multiple feedforward pathways. We recorded simultaneously from ganglion cells in the retina and cortical simple cells in the striate cortex with overlapping receptive fields and evaluated disynaptic connections with cross-correlation analysis. In all disynaptically connected pairs, the retinal receptive field center and overlapping cortical subregion always shared the same sign (either both $\mathrm{ON}$ or both $\mathrm{OFF}$ ). Connected pairs were similar in other respects, such as relative position and timing of their receptive fields, and thus obeyed the same rules of connectivity found previously for retinothalamic and thalamocortical connections. We found that a single RGC directly contributed on average to $\sim 3 \%$ of the activity of its cortical target. The relative timing of pairs of spikes from the retinal cell affected their efficacy in driving the cortical cell. When two retinal spikes were closely spaced $(<10$ $\mathrm{msec}$ ), the second spike was several times more likely to drive the cortical target. The relative magnitude of this disynaptic paired spike enhancement was considerably larger than has been found previously for retinogeniculate and geniculocortical connections. The amplified paired spike enhancement from retina to cortex ensures that signal transmission from retina to cortex is particularly effective when the retina fires a series of closely spaced action potentials.
\end{abstract}

Key words: simple cells; retinal ganglion cells; area 17; temporal summation; paired spike enhancement; disynaptic connections; synaptic efficacy; JPSTH; overlapping receptive fields; cross-correlation

\section{Introduction}

Connections in the pathway from retina to visual cortex in the cat are very precise. Single neurons in the lateral geniculate nucleus (LGN) of the thalamus generally receive strong input from one or two retinal ganglion cells (RGCs) and very weak input from a handful of others (Levick et al., 1972; Mastronarde, 1987; Usrey et al., 1999). Single simple cells in layer 4 receive convergent inputs from a very specific pool of at least 30 LGN cells (Reid and Alonso, 1995; Alonso et al., 2001). The functional influence of the retina on visual cortical neurons is therefore known in outline, but several factors make it quite difficult to infer from past studies the influence of a single RGC on the activity of simple cells. There are several times more LGN cells than there are RGCs (Levick et al., 1972), so that each RGC diverges onto a small pool of relay neurons. This causes fast $(<1 \mathrm{msec})$ and in some cases very strong synchrony in the LGN because of common input (Alonso et al., 1996; Usrey et al., 1998). Fast synchrony in the LGN is functionally significant because synchronous pools of LGN cells reconverge onto single cortical targets to drive them synergistically (Alonso et al., 1996, 2001). Because of this complex scenario, the combined effectiveness of multiple pathways en route to the

Received June 4, 2003; revised July 19, 2003; accepted July 21, 2003.

This work was supported by National Institutes of Health Grants R01 EY10115 and P30 EY12196. We thank Barry Lee for helpful discussions during the initial phase of this project, Sergey Yurgenson for many helpful suggestions and development of data analysis software, Kenichi Ohki, John Reppas, and Carrie McAdams for comments on this manuscript, and Sasha Vagodny for expert technical assistance with surgery and histology.

Correspondence should be addressed to Dr. Prakash Kara, Harvard Medical School, 220 Longwood Avenue, Boston, MA 02115. E-mail: pkara@hms.harvard.edu.

Copyright $\odot 2003$ Society for Neuroscience $\quad$ 0270-6474/03/238547-11\$15.00/0 cortex cannot be known unless one could record from all LGN cells that receive input from a given RGC, along with their target neuron in the cortex (Fig. 1).

To assess how well the firing of retinal spikes drives the responses of cortical cells, we recorded simultaneously from single neurons in the retina and visual cortex that had overlapping receptive fields. We used cross-correlation analysis to make a direct estimate of the efficacy of a single retinal neuron in evoking a spike in individual neurons in layer 4 of the cat primary visual cortex. Before our study, Lee et al. (1977) showed that disynaptic connections between RGCs and cortical simple cells can be robustly detected. However, their pioneering study did not address several issues that now warrant reinvestigation in the context of recent findings in the early visual system: (1) the dependence of connectivity on the detailed spatial and temporal receptive field properties of presynaptic and postsynaptic neurons (Alonso et al., 2001) and (2) modulation of postsynaptic responses based on the temporal patterns of presynaptic firing.

That successive retinal spikes are increasingly effective in driving LGN responses was first demonstrated by Mastronarde (1987) and has since been examined in greater depth by others (Levine, 1998; Usrey et al., 1998; Levine and Cleland, 2001; Rowe and Fischer, 2001; Weyand, 2001). Similar "paired spike enhancement" occurs for monosynaptic connections between LGN and visual cortex (Usrey et al., 2000). One of the goals of the current study was to examine the extent to which the disynaptic influence of an RGC on a cortical neuron might be affected by the recent activity of the RGC. We found not only that paired spike enhancement exists for the disynaptic connection but also that it 


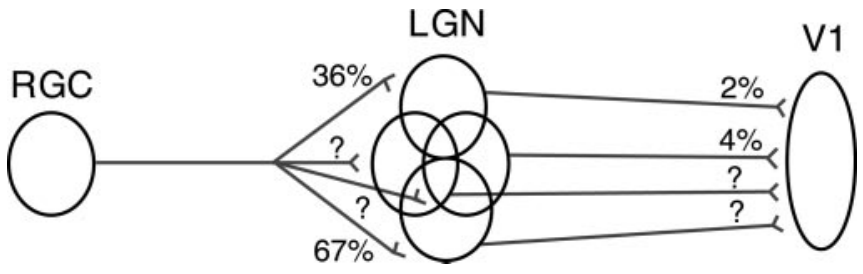

Figure 1. Deducing retinocortical strength from monosynaptic retinothalamic and thalamocortical connections. The divergence from a single RGC to multiple thalamic (LGN) cells and subsequent reconvergence to single cortical cells (V1) results in the need to account for multiple pathways, each with different strengths. Contribution (as defined in Materials and Methods) is used to measure the strength of monosynaptic connections. The RGC-LGN contributions shown are from an actual "triplet" recording: one RGC to two LGN cells (36\%, 67\%) (Usrey et al., 1998, 1999). Similarly, triplets with two LGN inputs to one cortical cell have been studied (Reid and Alonso, 1995; Alonso et al., 1996) (contributions of 2\%, 4\% are typical of geniculocortical connections). Even with these recordings, other pathways from a single RGC are not accounted for (question marks). Simultaneous recordings from a single RGC and a single cortical simple cell are technically straightforward and provide a direct estimate of the effectiveness of a single retinal afferent in driving a cortical simple cell.

was stronger than might be expected from past studies of monosynaptic connections.

\section{Materials and Methods}

Preparation and anesthesia. Experiments were performed on 25 anesthetized and paralyzed adult cats. Our surgical and electrophysiological methods have been described in detail elsewhere (Kara et al., 2000, 2002). Surgical anesthesia was induced with ketamine $\mathrm{HCl}(10 \mathrm{mg} / \mathrm{kg}$, i.m. $)$ and continued with thiopental sodium $(20 \mathrm{mg} / \mathrm{kg}$, i.v., supplemented as needed). All pressure points and incision sites were treated with a topical anesthetic ( $2 \%$ lidocaine $\mathrm{HCl}$ ). The electroencephalograph was measured over the frontal lobes to monitor the depth of anesthesia. A craniotomy was made over the left hemisphere of the brain above the primary visual cortex (area 17). A metal ring was glued to surgically exposed lateral sclera of the right (contralateral) eye and attached to a post to prevent small eye drifts that may otherwise occur even with adequate anesthesia and paralysis. The metal ring also facilitated the insertion of an intraocular recording electrode (see below). A fiber-optic light source was used to reflect retinal landmarks onto a tangent screen placed $114 \mathrm{~cm}$ in front of the eyes. Optical refraction was achieved with gas-permeable contact lenses such that surface retinal blood vessels were focused on the tangent screen.

After all surgical procedures were completed, anesthesia was maintained with thiopental sodium $\left(2-3 \mathrm{mg} \cdot \mathrm{kg}^{-1} \cdot \mathrm{hr}^{-1}\right.$, in $0.9 \%$ saline, i.v.). Paralysis was achieved by vecuronium bromide (0.2-0.3 $\mathrm{mg} \cdot \mathrm{kg}^{-1} \cdot \mathrm{hr}^{-1}$, in $0.9 \%$ saline with $5 \%$ dextrose, i.v.). Animals were mechanically ventilated and expired $\mathrm{CO}_{2}$ was regulated at $3.8-4.2 \%$. All surgical and experimental procedures were in accordance with National Institutes of Health and United States Department of Agriculture guidelines and were approved by the Harvard Medical Area Standing Committee on Animals.

Electrophysiology, visual stimulation, and receptive fields. Simultaneous recordings from RGCs and simple cells in layer 4 of the striate cortex were made with platinum-plated tungsten-in-glass electrodes (Merrill and Ainsworth, 1972). Retinal and cortical electrodes were positioned so that their receptive field centers shared similar eccentricity (within $5^{\circ}$ of the area centralis) and were completely or partially overlapped with one another (see Results, Fig. 2).

Each experiment began by first searching for simple cells along the medial bank of the striate cortex. Our strategy for targeting layer 4 has been described in detail (Kara et al., 2002). Layer 4 simple cells were identified as having at least one ON and one OFF spatially segregated subfield. The retinal recording electrode was targeted via a rotating $x-y-z$ stage and a rotating microelectrode carrier.

Receptive fields were characterized via visual stimuli displayed on a 15 inch Nokia Multigraph 447X (128 Hz frame rate) or in later experiments on a 21 inch Viewsonic 817P monitor $(170 \mathrm{~Hz}$ frame rate) at a distance of
$114 \mathrm{~cm}$ from the animal's eyes. A binary white noise stimulus was used to obtain spatiotemporal receptive-field maps, as described previously (Reid et al., 1997). Individual stimulus pixels were $0.4-0.8^{\circ}$ wide so that cortical receptive field subregions were at least four to eight pixels long and two to three pixels wide. The white noise stimulus was updated every $15.6,31.3$, or $29.4 \mathrm{msec}$ (two or four frames at a $128 \mathrm{~Hz}$ frame rate or five frames at $170 \mathrm{~Hz}$ ). The receptive fields were calculated by correlating each spike with the stimuli that preceded it at various delays between stimulus and spike. Although the stimuli had different durations in different experiments, the receptive fields were calculated with a $15 \mathrm{msec}$ time bin. The " $0 \mathrm{msec}$ " bin began with the time of the spike and extended back in time 15 msec.

During the experiments, receptive field maps were used exclusively for assessing the degree of spatial overlap between the simultaneously recorded retinal and cortical receptive fields. For assessing functional connectivity, $4 \mathrm{~Hz}$ drifting sinusoidal gratings of $50 \%$ contrast were used to drive the retinal and cortical neurons strongly to aid in cross-correlation analysis. The orientation of the grating was matched to the optimal orientation of the simple cell. Between 800 and 20,000 cycles of the grating stimulus were presented for each recording. Direct intraocular recordings of single retinal ganglion cells of high signal-to-noise ratios for extended periods $(30-120 \mathrm{~min})$ were attained using a narrow-diameter, high-impedance Ainsworth electrode mounted on a customized miniature Narishige (Tokyo, Japan) MO-95 hydraulic micromanipulator. A combination of modified phase tests (Hochstein and Shapley, 1976), comparison of neighboring RGC receptive field sizes in any given experiment, and comparison of the RGC center to the simple cell subfield diameter in each pair (see Fig. $4 B$ ) suggested that our sample of RGCs consisted of both X and Y classes. However, RGCs from functionally connected disynaptic retinal-cortical pairs were invariably from the X-cell class, consistent with a previous report (Lee et al., 1977). To maximize our chance of finding rare disynaptic connections, we consistently targeted our retinal and cortical electrodes close to area centralis so that our retinal sample was biased toward recording from X-cells.

Data collection. Neural signals were amplified with a head stage-based AC amplifier (model 1800; A-M Systems, Carlsborg, WA), bandpassfiltered between 0.3 and $5 \mathrm{KHz}$, and digitized at $20 \mathrm{KHz}$ using Discovery data collection software and hardware (Datawave Technologies, Longmont, CO) or, in later experiments, Power 1401 hardware and Spike2 software (Cambridge Electronic Design). All data were reanalyzed offline to confirm single-unit isolation via waveform analysis, cluster cutting, and the presence of an absolute refractory period in the autocorrelogram (for examples of retinal and cortical single-unit isolation, see Kara et al., 2000).

For RGC data, second units on a single electrode constituted only 4 of 252 cases. For our cortical recordings, 7 of 123 simple cells were second units. We only used second units that fired out of phase with the primary unit because the receptive fields were of opposite sign (OFF vs ON) or completely spatially offset. Thus, when we resolved two well isolated units from a single electrode, the recording was free of contamination by spikes that could have otherwise fired in synchrony.

Analysis of disynaptic cross-correlations. White noise visual stimuli drove cortical simple cells relatively poorly, at an average rate of $\sim 3 \mathrm{~Hz}$ (mean \pm SEM, $2.93 \pm 0.31 ; n=123$ simple cells) versus RGCs (55.25 \pm $1.85 ; n=252$ RGCs). However, sine gratings drive RGCs, LGN cells, and primary visual cortex (V1) simple cells quite strongly (Kara et al., 2000), making it easier to collect a sufficient number of spikes for correlation analysis. Thus, to assess the presence of a functional connection, the cross-correlogram peak was analyzed from RGC-V1 spike trains during visual stimulation with $4 \mathrm{~Hz}$ drifting sine gratings. The slow stimulusdependent component (see Fig. $2 A, B$, red curve) was isolated from the raw correlogram (see Fig. $2 A, B$, blue curve) by shifting the target (cortical) spike train by one stimulus cycle or $\sim 250 \mathrm{msec}$ (shift predictor method, as described by Perkel et al., 1967; Epping and Eggermont, 1987). Because the response to sine gratings of both RGCs and V1 simple cells in cortical layer 4 are stationary with very low (sub-Poisson) cycleto-cycle spike count variability (Kara et al., 2000), residual correlations after subtraction of the shift predictor from the raw correlogram accurately reflected the fast and pure "neural" correlation (see Fig. $2 A, B$, 
green traces). In our first-order correlograms, the retinal spike train is the reference spike train, and retinal spikes occur at time 0 . The correlogram thus represents the probability of a cortical spike occurring at various delays after a retinal spike. To quantify the strength of the functional disynaptic connection, we calculated the number of spikes in the peak of the shift-corrected correlogram (correlogram range, -50 to $+50 \mathrm{msec}$; bin width, $0.5 \mathrm{msec}$; as in Fig. 2). The peak magnitude was defined as the sum of bin values from -1 to $+3 \mathrm{msec}$ around the bin with the highest count (constrained to +1 to $+10 \mathrm{msec}$ in the cross-correlation; peak times actually ranged from 4.5 to $9 \mathrm{msec}$ ). The highest magnitude bin was required to be $>3 \mathrm{SD}$ above baseline noise for a significant functional connection (see Fig. 2, dotted horizontal lines). Connection strength was quantified as efficacy (peak magnitude normalized by the number of reference or retinal spikes) and contribution (peak magnitude normalized by the number of target or cortical spikes; Levick et al., 1972). Thus, efficacy is the percentage of RGC spikes that are followed by a cortical spike within 5-9 msec, and contribution is the percentage of cortical spikes that are preceded by an RGC spike within a time window of 5-9 msec. Because retinal ganglion cells always had higher mean firing rates than cortical cells, any given pair typically had a higher contribution than efficacy (see Fig. 3).

Although the shift-corrected procedure has been used widely in past studies, recent studies from our laboratory (Usrey et al., 1999; Alonso et al., 2001) have used an alternate method, primarily to facilitate an evaluation of connection strength from spike trains collected during the presentation of a nonperiodic visual stimulus (white noise). This method removes the slow baseline by high-pass filtering the correlogram, so that only the fast peak remains. Here, we used a slightly modified version of this method in which the peak (if any, $>3$ SD above baseline) was first detected and then removed by interpolating the data in the range of -1 to $+3 \mathrm{msec}$ around the peak. The resultant correlogram, with the peak deleted, was then low-pass-filtered at $75 \mathrm{~Hz}$ to provide an estimate of the baseline, which was then subtracted from the raw cross-correlogram. Peak area, efficacy, and contribution were then calculated in the same manner as for the shift predictor correction method, described in the previous paragraph. Examples of filter-subtracted correlograms are shown in supplementary Figure 1 (available at www.jneurosci.org).

For the purpose of avoiding false negatives attributable to a lack of a sufficient number of spikes, our sample excluded raw negative correlograms (no disynaptic peaks) that had $<50$ spikes in the baseline, defined here as the period from -4.0 to $0.0 \mathrm{msec}$ (Reid and Alonso, 1995) (see Results).

Analysis of receptive field overlap. The spatial receptive field was defined as the average stimulus that preceded each spike by $15-90 \mathrm{msec}$ (Alonso et al., 2001; Kara et al., 2002). If the response changed sign (rebounded), only frames before the rebound were used. Before averaging, each frame of the $16 \times 16$ map was resampled to $256 \times 256$ pixels (via linear interpolation) to minimize binning artifacts during Gaussian fitting of receptive field centers of RGCs and both subfields of simple cells (one ON and one OFF subfield). RGC center size and location were quantified by finding the best-fitting circular Gaussian (as defined by Usrey et al., 1999). These fits are indicated with a circle drawn at $1.75 \sigma$, which correspond to $4.7 \%$ of the peak (see Fig. $2 A, B$, yellow circle). The spatial extents of the two strongest cortical simple subfields were each specified via elliptical Gaussians (as defined by Kara et al., 2002). The subfields are indicated in Figure 2, $A$ and $B$, white ellipses, also drawn at $4.7 \%$ of the peak values.

The relative position between the RGC and cortical simple cell was evaluated using the normalized dot product of the two spatial receptive fields (Alonso et al., 2001), where the pixels of the RGC and V1 receptive fields are shifted to obtain a dot product of the largest absolute value. The original scalar dot product of the two receptive fields divided by this absolute value dot product is defined as relative overlap (see Fig. 4A). A value of +1 denotes the best relative position with the same sign; 0 , no overlap; and -1 , best overlap but opposite sign (e.g., ON-center RGC over OFF subfield of simple cell). Because RGCs and cortical simple cells inherently always have very different receptive field sizes and shapes (see Fig. 2), this normalization proved the most informative (Alonso et al., 2001).
Typically, the RGC center overlapped primarily with a single cortical simple cell subfield (see Fig. 2). To prevent ambiguity in comparing receptive field width in pairs in which the RGC receptive field center overlapped extensively with both subfields of the cortical cell (particularly for Y-like RGCs, which have larger receptive field centers), the cortical subfield used to compare receptive field width was always the subfield that shared a larger area (intersection) of overlap with the RGC.

Time course of receptive field. The frame-by-frame evolution of the spatial receptive field common to both the RGC and V1 simple cell (spatially overlapping and same sign) were quantified according to the methods described in detail by Alonso et al. (2001). Such impulse responses (or temporal weighting functions) of common pixels (see Fig. $5 C)$ were constructed from the raw $(16 \times 16$ grid $)$ receptive field maps (see Fig. 5B). The RGC-V1 differences in the time-to-peak and zero crossing time of the impulse response were compared for all pairs, both connected and unconnected (see Fig. 6). The bin width for all impulse responses was set at $15 \mathrm{msec}$. Reported peak and zero crossing times did not necessarily correspond to multiples of the $15 \mathrm{msec}$ bin time because values were derived from cubic spline-interpolated impulse response functions.

Comparing efficacies of first versus second retinal spikes. For any given RGC-V1 pair, all RGC and V1 spikes were used in the analysis of the existence [via a fast neural peak in the cross-correlogram (Pos Xcorr)] or absence [no significant peak in cross-correlation (Flat Xcorr)] of a functional disynaptic connection. To examine whether the pattern of preceding retinal activity influenced the efficacy of a retinal spike in driving a cortical target to fire, we examined pairs of retinal spikes with different interspike intervals. Each pair of retinal spikes had to be preceded by a minimum period of quiescence to ensure an approximately equivalent level of baseline activity immediately preceding all first retinal spikes (see Fig. 7, compare $A, B$ ). This dead time (DT) was set at $10 \mathrm{msec}$ for all data presented in this paper, although larger $(20 \mathrm{msec})$ and smaller $(5 \mathrm{msec})$ windows were analyzed and found to yield qualitatively similar results. Each pair of retinal spikes had an interspike interval (ISI) of 4-30 msec. The efficacy of the first and second retinal spikes were calculated as described above in Analysis of disynaptic cross-correlations (shift predictor subtraction and spike counts in peak divided by counts in the retinal spike train) and plotted for all retinal paired spike ISIs (see Fig. 8). We emphasize that comparison between first and second retinal spikes for retinal ISIs greater than the DT (see Fig. 8, dashed lines) may be difficult to interpret. This is because the first retinal spike in the pair might be preceded by a shorter interval than the second retinal spike, which is the reverse of the situation for shorter ISIs. Also, paired spike enhancement should not be confused with priority transmission via bursts. Using the classic phenomenology of bursts [two to eight spikes separated by intervals of $<4 \mathrm{msec}$, preceded by an interval of at least $100 \mathrm{msec}$ without spiking (Lu et al., 1992)], such events are not detected in RGCs. Even if the requirement of the interval of quiescence is not invoked, RGCs typically do not fire action potentials that are spaced by $<4 \mathrm{msec}$ (see supplementary Fig. 2, available at www.jneurosci.org).

Normalized joint peristimulus time histograms (JPSTHs) (see Fig. $10 B)$ were calculated as described by Aertsen et al. (1989, their equation 9, calculated using the data analysis program Neuroexplorer from $\mathrm{Nex}$ Technologies, Littleton MA). These functions are presented as twodimensional plots, each pixel of which represents a correlation coefficient that can theoretically range from -1 to +1 . JPSTH pixels close to the diagonal (see Fig. $10 \mathrm{~B}$, yellow, orange, red pixels) reflect correlations induced by neural connection as a function of the timing of the responses relative to the visual stimulus. The JPSTH was used to evaluate whether paired spike interactions may be attributable to ascending or descending baselines in the cross-correlogram, that is, attributable to higher or lower relative cortical firing rates at approximately the time of first and second retinal spikes.

\section{Results}

We studied 284 pairs of simultaneously recorded retinal ganglion and cortical simple cells. An individual simple cell was often recorded with multiple retinal ganglion cells sequentially; our 284 
pairs consisted of 252 unique retinal cells and 123 unique simple cells.

To avoid false negatives in estimating the fraction of connected pairs, we excluded negative correlograms if they had $<50$ spikes in the baseline in the period from -4.0 to $0.0 \mathrm{msec}$ (Alonso et al., 2001). This is of concern particularly when the two overlapping receptive fields in a pair have opposite polarity (ON vs OFF) because their firing would be anticorrelated. The analysis presented in this paper is thus limited to 116 of 284 pairs. In these pairs, we found statistically significant functional connections in $12 \%$ of cases (14 of 116).

We present results to confirm that functional connections between retinal ganglion cells and cortical simple cells (separated by two synapses) can be reliably detected. The strength of disynaptic functional connections is quantified using the spike efficacy and contribution measures described in $\mathrm{Ma}$ terials and Methods. Then the dependence of connectivity on various receptive field properties of the simultaneously recorded pair is compared. Finally, we examine whether the relative timing of successive pairs of retinal spikes modulates the strength of disynaptic connections.

\section{Detection and strength of disynaptic connections}

Two examples of functionally connected retinocortical pairs are shown in Figure 2,

$A$ and $B$. The first example shows an ON-center X-type RGC overlapping with the elongated $\mathrm{ON}$ subfield of a cortical simple cell (Fig. $2 \mathrm{~A}$ ), and the second pair is from an OFF-center X-type RGC overlapping with an OFF subfield of another simple cell (Fig. $2 B$ ). The contributions of the two connections (assessed from the magnitude of the peak of the shuffle-subtracted correlogram; Fig. $2 A, B$, green curve) were 2.2 and $1.9 \%$, for Figure 2, $A$ and $B$, respectively. The average contribution and efficacy over all connected pairs were 3.0 and $1.1 \%$, respectively (Fig. $3 A$ ). The latencies to the peak bin in the first-order cross-correlograms were all in the range of 4.5-9.0 msec (mean \pm SEM, $6.7 \pm 0.3$ msec; $n=14$ pairs), consistent with a disynaptic pathway (for retina-cortex latency, see Lee et al., 1977) (for 2-4 msec latency from LGN-cortex, see Alonso et al., 2001). The strength of the connection depended on the relative overlap of the simultaneously recorded RGC and V1 receptive fields, such that perfectly overlapping receptive fields generally were associated with the strongest connections (Fig. 3B).

To test the fidelity of our efficacy and contribution estimates via shift-corrected correlation peaks (described above), we used another "filter" method of extracting the neural peak from the raw cross-correlograms (see Materials and Methods). Across all connected pairs, the mean contribution and efficacy between the two independent measures were indistinguishable: i.e., shift-corrected efficacy $=1.1 \pm 0.3$; filtered efficacy $=0.9 \pm 0.3($ mean \pm SEM $) ; p=$ 0.73 (Mann-Whitney $U$ test); and shift-predictor corrected contribution $=3.0 \pm 0.4$; filtered contribution $=3.1 \pm 0.5$ (mean \pm $\mathrm{SEM}) ; p=0.85$ (Mann-Whitney $U$ test).
V1 receptive field
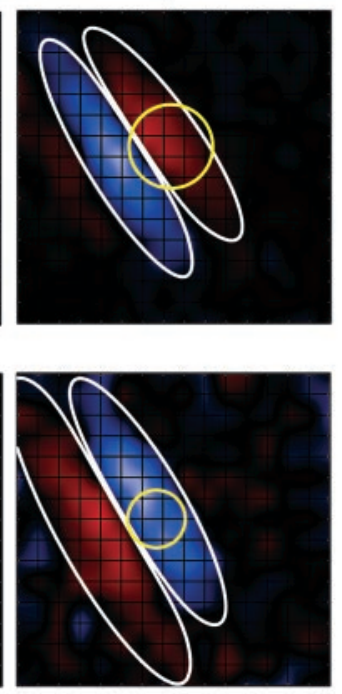

RGC-V1 cross-correlation
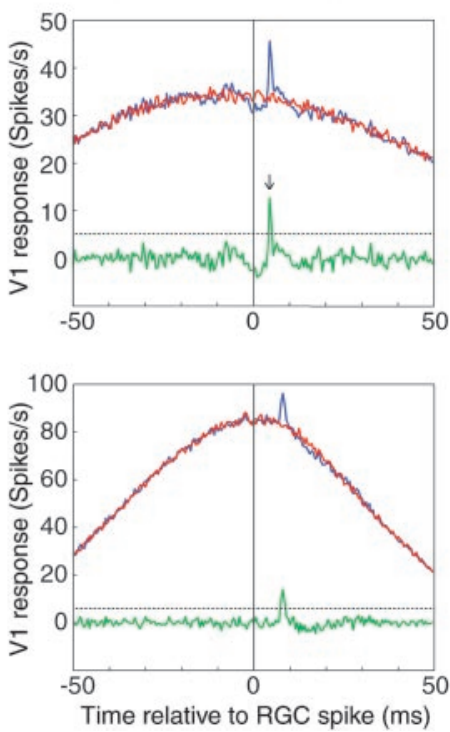

50
Figure 2. Receptive fields and cross-correlograms for two functionally connected retinocortical pairs. $A, 0 \mathrm{~N}$-center spatial receptive field of an $\mathrm{RGC}$ overlapping with the $0 \mathrm{~N}$ subfield of a cortical simple cell in striate cortex (V1). $0 \mathrm{~N}$ pixels are shown in red, and OFF pixels are shown in blue. Grid lines show individual stimulus pixels $\left(0.75^{\circ}\right)$. The relative overlap of the two receptive fields (black arrow) had a latency of $4.5 \mathrm{msec}$. Efficacy and contribution were 1.1 In total, 107,785 RGC and 90,358 V1 spikes were collected in 56 min of grating stimulation. The dotted horizontal black line in each cross-correlogram represents the significance criterion for the fast neural peak, i.e., 3 SDs above the baseline.

\section{Receptive field properties in functionally connected pairs} Primary cortical subfield

In only 1 of 14 pairs did the RGC receptive field center overlap with the weaker of two cortical subfields (Fig. $2 A$ ). In all other cases, functional connections were detected only when the RGC receptive field center overlapped with the strongest cortical subfield (Fig. 2) (see supplementary Fig. 1, available at www.jneurosci.org).

\section{Receptive field sign and position}

Functional connections (Pos Xcorr) were detected only when the RGC center and its corresponding cortical subfield were of the same sign (ON-center RGC with ON subfield of simple cell, $n=$ 5 of 14 pairs; OFF-center RGC with OFF subfield of simple cell, $n=9$ of 14 pairs). Furthermore, good overlap between the RGC receptive field center and the $\mathrm{V} 1$ subfield was necessary for detecting functional connections: we found that the relative overlap of the two receptive fields was between 0.6 (moderate overlap) and 1.0 (best possible overlap) for all but one connected pair (Fig. $4 A$, filled bars). In no case was a connected pair detected that had a negative relative overlap (opposite sign) (Fig. $4 A$, open bars).

\section{Receptive field width}

To assess whether the relative receptive field size was a determinant of functional connectivity, we compared the width of each RGC center to the width of the overlapping cortical subfield. We found that the ratio of receptive field widths for connected and unconnected pairs had a very similar distribution (Fig. $4 B$ ), so it was not a predictor of disynaptic connectivity. The relative size of receptive fields was also found to be the weakest predictor of connectivity between LGN and visual cortex (Alonso et al., 2001). 

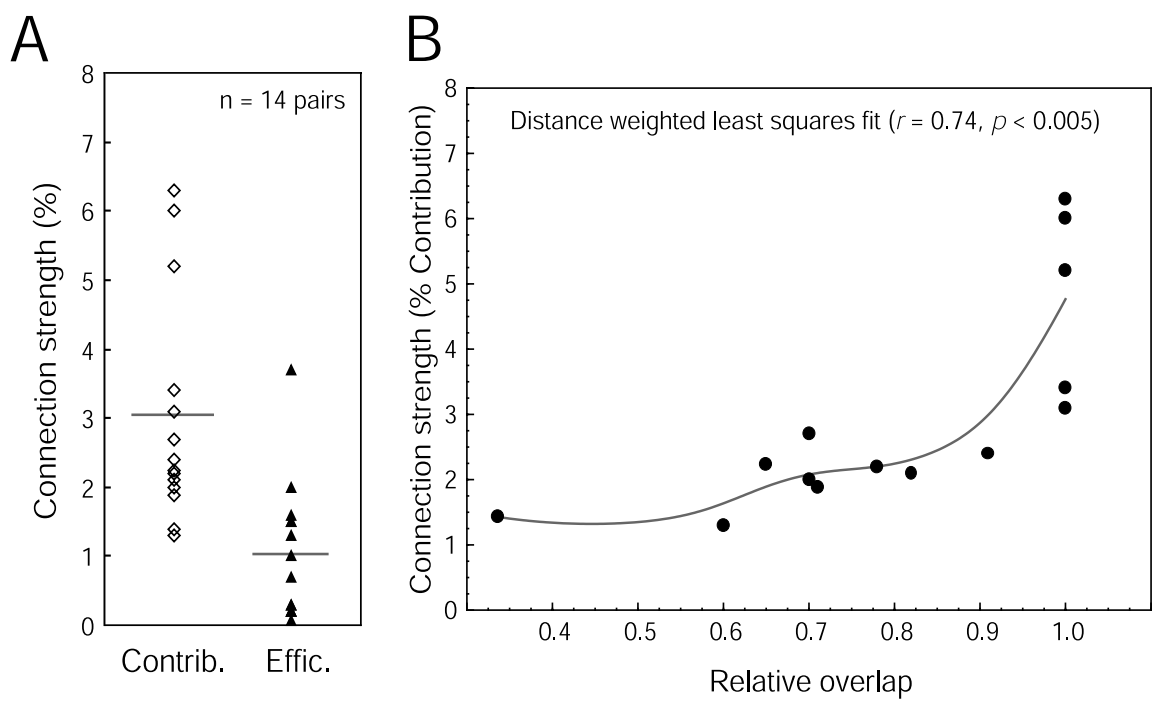

Figure 3. Summary distributions of contribution, efficacy, and relative overlap in connected retinocortical pairs. $A$, Contribution (Contrib., open diamonds) and efficacy (Effic., filled triangles) ranged from 1.2 to $6.2 \%$ and 0.2 to $3.6 \%$, respectively. Means are shown as horizontal gray lines. $B$, The connection strength was strongest when the overlap of RGC and V1 receptive fields was perfect (relative RGC-V1 overlap = 1). However, perfect overlap did not guarantee the existence of a functional connection because 11 pairs with perfect overlap did not display statistically significant neural peaks via cross-correlation analysis.

\section{Receptive field timing: peak and zero crossing time}

Receptive field maps evolve over several tens of milliseconds (Reid et al., 1997), so that each region of a receptive field has a characteristic time course. It was previously found that relative timing differences between thalamic and cortical cells influenced the likelihood of finding a connection (Alonso et al., 2001). It has been postulated that a dependence of connectivity and timing might be related to the distinction between lagged and nonlagged cells in the LGN (Saul and Humphrey, 1992). Because lagged cells exist only in the LGN and not in the retina, we therefore asked whether a similar relationship between connectivity and timing might be found between retina and cortex. Examples of an RGC and a $\mathrm{V} 1$ receptive field averaged over two stimulus frames are shown in Figure 5, $A$ (interpolated at $256 \times 256$ pixels; see Materials and Methods) and $B$ (raw, $16 \times 16$ pixels). We examined the entire time course of the receptive fields for pixels that were common to both the retinal and cortical receptive fields (Fig. 5B, four common ON pixels in yellow boxes). The normalized time courses (impulse responses) of the RGC and $\mathrm{V} 1$ receptive fields (for the same pair shown in Fig. 5A,B) are shown in Figure 5C. Both impulse responses peaked at $23 \mathrm{msec}$, but the V1 impulse response was more prolonged (later zero crossing time). We quantified the peak and zero crossing times in 43 pairs ( 14 connected and 29 unconnected) in which each pair had a sufficient number of overlapping pixels that were of the same sign (ON or OFF; see Materials and Methods). The distributions for peak time were indistinguishable for connected (Pos Xcorr) versus unconnected (Flat Xcorr) groups (Fig. 6A). However, the likelihood of detecting connections was $>1.5$ times higher when the difference in zero crossing time was $<20 \mathrm{msec}$ (Fig. $6 B$ ).

\section{Efficacy of first versus second retinal spikes}

Thus far, we have examined how the probability of finding a disynaptic functional connection depends on receptive-field properties (sign, size, width, and time course) of a simultaneously recorded RGC and V1 cell pair. We used a first-order correlation analysis that yields an overall connection strength averaged over all spikes, and thus ignores possible dynamics of functional con- nectivity. A previous study of functional retinogeniculate connections reported that efficacy is modulated by the temporal pattern of spiking in the presynaptic neuron, such as with paired spike interactions in both X- and Y-cells (Usrey et al., 1998). For retinogeniculate and geniculocortical connections, pairs of presynaptic spikes having short ISIs are much more effective in driving a postsynaptic target than spikes with longer ISIs. In this section, we evaluate whether such interactions between pairs of RGC spikes could be detected across the disynaptic pathway.

To evaluate the interaction between pairs of retinal spikes in modulating retinocortical efficacy, one must have a greater number of spikes than is needed for the detection of those functional connections. This is because for the interaction analysis, the retinal spike train is filtered for pairs of spikes of short interspike intervals (4-30 msec) preceded by quiescent periods of at least $10 \mathrm{msec}$ (the dead time; see Materials and Methods) (Fig. $7 A, B)$. Empirically, we found that a minimum of 20,000 retinal spikes was required for paired spike analysis of sufficient signal-to-noise ratio. Eight connected pairs satisfied these criteria, with an average of $\sim 100,000$ retinal spikes and $\sim 30,000$ cortical spikes.

An example illustrating the differences in overall efficacy and the efficacy of first versus second retinal spikes is shown in Figure 7. Cross-correlograms of two RGC-V1 pairs are shown in Figure $7 C-F$. The first pair showed a particularly strong connection (contribution, 4.7\%; efficacy, 2.7\%) (Fig. 7C), whereas the second pair displayed a much weaker connection (contribution, 2.2\%; efficacy, 1.1\%). Filtering the retinal spike train for pairs of spikes revealed that the second retinal spike was consistently more effective than the first retinal spike in driving cortical neurons. An example in which the interval between the pair of retinal spikes was $10 \mathrm{msec}$ is shown in Figure $7 E$. The occurrence of the first retinal spike in this paired spike correlogram is at time 0 (first dashed line). The second retinal spike is depicted by the second dashed line $10 \mathrm{msec}$ later. The efficacy of the second spike was twice as large as the efficacy of the first spike. A similar analysis for another retinocortical pair is shown in Figure $7 F$. In this case, when the interval between the two retinal spikes was restricted to $5 \mathrm{msec}$, the second retinal spike was threefold more effective than the first retinal spike.

Interaction of pairs of retinal spikes on cortical firing was calculated for a range of intervals $(4-30 \mathrm{msec})$ (Fig. $8 A$ ). The efficacy of first versus second spikes from a single pair using this entire range of retinal paired spike intervals is shown in Figure $8 B$. In this case, the enhancement of the second retinal spike is very transient, lasting $5 \mathrm{msec}$, after which the efficacies of both first and second retinal spikes are virtually identical. The average response from eight pairs are shown in Figure $8 C$. Note that the efficacy of the first retinal spike remains uniform at all ISIs (oneway ANOVA, $p=0.89$ ) suggesting that imposing a $10 \mathrm{msec}$ dead time before each retinal spike in the pair provided a satisfactory baseline for paired spike comparisons. The efficacies of second spikes were not uniform across the range of ISIs tested (one-way ANOVA, $p<0.005$ ). We found that second retinal spikes were 
A Receptive-field position
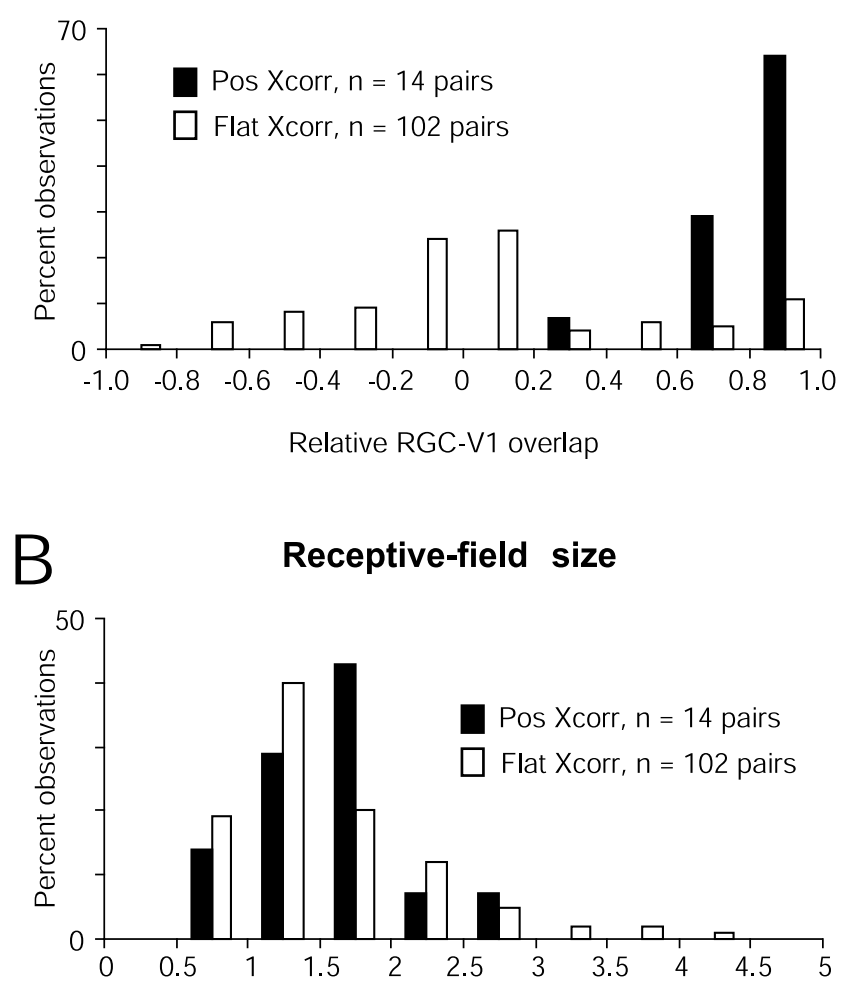

(RGC center/V1 simple cell subfield) width

Figure 4. Distributions of connected versus unconnected RGC-V1 pairs for relative position $(A)$ and size $(B)$ of $R G C$ and $V 1$ receptive fields. Existence of functional connections determined by the presence of significant neural peaks in cross-correlograms depicted as filled bars (Pos $X$ Corr) and absence of connections depicted as open bars (Flat Xcorr). Negative relative overlaps in $A$ represent overlapping $\mathrm{RGC}-\mathrm{V} 1$ receptive field pairs of opposite signs (ON vs OFF). In no cases were connections detected in such instances of opposite-sign overlap. With the exception of one pair, functional connections were detected when overlap ranged from +0.6 to +1 . The distributions for relative field position between Pos Xcorr and Flat XCorr were significantly different ( $p<0.02$, Kolmogorov-Smirnov test). The distributions of relative receptive field widths in connected versus unconnected pairs $(B)$ were indistinguishable $(p=0.32$, Kolmogorov-Smirnov test). In this and subsequent figures, distributions are plotted as percentage observations rather than the number of pairs because the sample sizes of connected (14 of 116) and unconnected (102 of 116) pairs were vastly different. Thus, in $A, 65 \%$ of Pos Xcorr for a relative position of $0.8-1.0$ represents 9 of 14 connected pairs, and $11 \%$ of Flat Xcorr at the same relative position represents 11 of 102 unconnected pairs.

more effective than first retinal spikes (nested ANOVA, $p<$ $0.005)$ for pairs of retinal spikes that ranged from 4 to $9 \mathrm{msec}(t$ tests for each $1 \mathrm{msec}$ bin, $p<0.05$ for all 5 bins). Pairs of retinal spikes that had ISIs of 10-30 msec produced indistinguishable first and second paired spike efficacies ( $t$ tests, $p=0.2-0.9$ ). The narrow window of the paired spike effect is consistent with a simple postsynaptic biophysical mechanism of temporal summation (Usrey et al., 2000; Weyand, 2001).

Paired spike interactions: control for ascending baseline in the cross-correlogram

It is known that cross-correlation strength can be strongly modulated by an external stimulus (Aertsen et al., 1989), so it was important to ensure that the paired spike effect was not an artifact caused by the $4 \mathrm{~Hz}$ drifting grating we used to evoke spikes. In general, the receptive fields of disynaptically connected neurons are well overlapped so that their responses tend to occur at approximately the same time (Fig. $9 A$, left). In this case, the baseline
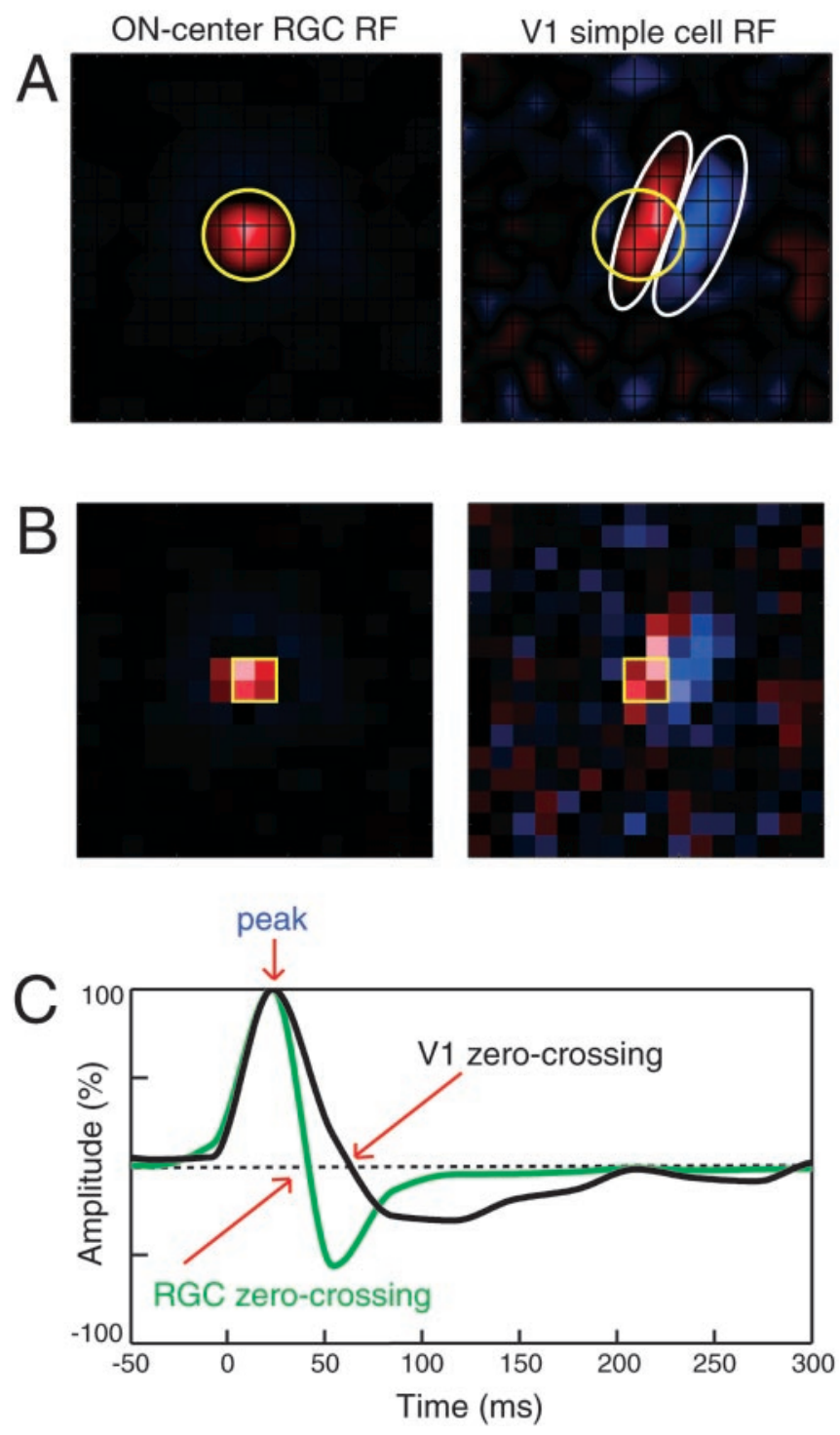

Figure 5. Time course of receptive fields from a disynaptically connected ON-center RGC and a cortical simple cell. $A$, Smoothed spatial receptive field maps ( $256 \times 256$ pixels, linearinterpolated; see Materials and Methods). $B$, Raw maps ( $16 \times 16$ pixels) used to generate smoothed maps in $A$ and to compute response time courses of the four $0 \mathrm{~N}$ pixels ( $B$, within yellow squares) that are common to both the RGC and V1 cells. Grid lines show individual stimulus pixels $\left(0.75^{\circ}\right)$. C, Impulse responses, or response time courses, of an RGC (green curve) and V1 cell (black curve). Both responses peaked at the same time ( $23 \mathrm{msec}$ ), but the zero crossing time of the $\mathrm{V} 1$ response is $22 \mathrm{msec}$ slower than the RGC.

of the cross-correlogram between the two neurons is usually flat (Figs. 7D,F, 9B, left). However, if the responses of the two neurons are phase-shifted so that the $\mathrm{V} 1$ response (Fig. $9 A$ right, gray curve) occurs later than the RGC response (black curve), this generally leads to a cross-correlogram in which the baseline is ascending rather than flat (Figs. $7 C, E, 9 B$, right). In this case, paired spike enhancement on an ascending baseline (Fig. 7E) could simply be an artifact of the higher cortical firing rate at the time of the second spike (Fig. 9B, right).

If the apparent paired spike enhancement were attributable to such a stimulus-dependent mechanism, we would expect that the efficacy of the first and second spikes would remain different for a broad range of retinal ISIs (rather than converge, as in Fig. $8 B, C)$. In one connected pair, the efficacy of the second spike remained higher than that of the first spike for all retinal paired 


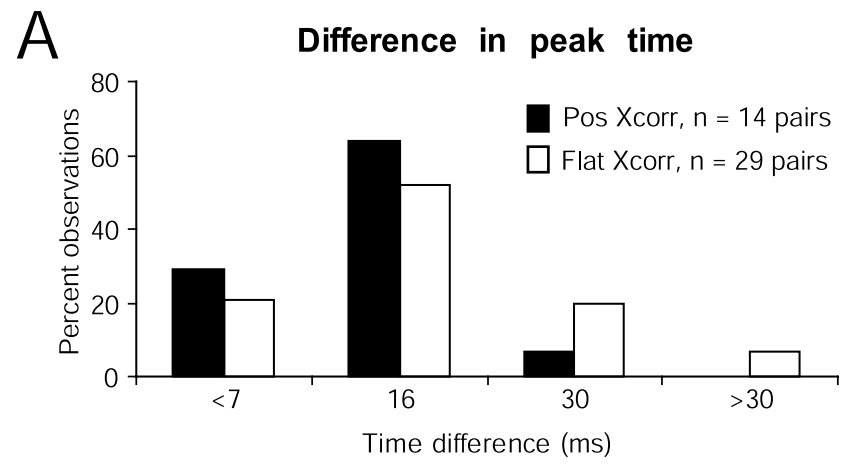

B

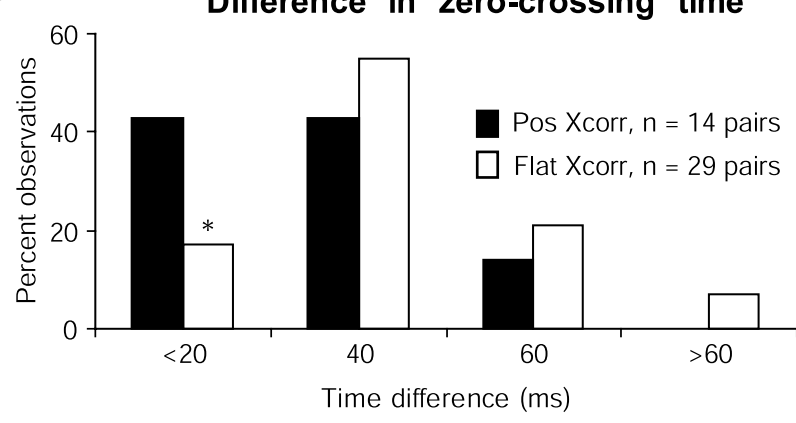

Figure 6. Distributions of peak and zero crossing time differences in connected versus unconnected RGC-V1 pairs. Difference in peak time $(A)$ and difference in zero crossing time $(B)$ distributions are statistically indistinguishable in connected (Pos Xcorr) and unconnected (Flat Xcorr) pairs ( $p=0.99$, Kolmogorov-Smirnov tests). However, when the zero crossing time is very similar in RGC and V1 cells ( $<20$ msec difference), there was a significantly higher incidence of detecting functional connections $\left({ }^{*} p<0.05\right.$, difference between two proportions test) (Blalock, 1972).

spike intervals up to $30 \mathrm{msec}$ (Fig. 10A). This example corresponds to the cross-correlogram with the ascending baseline shown in Figure 7E. The responses of these data are shown in Figure $10 \mathrm{~B}$ as PSTHs from time 0 to $130 \mathrm{msec}$. The RGC PSTH is much wider than the cortical PSTH, and the cortical response peaks toward the end of the retinal response.

To analyze the dependence of the correlation strength on the visual stimulus, we calculated the normalized JPSTH (Fig. $10 \mathrm{~B}$ ). Although the correlograms in Figures 2 and 7 allow us to infer dependence of cortical spikes on retinal firing on average, the JPSTH illustrates how this correlation between retina and cortex changes for different phases of the visual stimulus. Each bin in the JPSTH indicates the joint probability that a retinal spike ( $y$-axis) and a cortical spike ( $x$-axis) occurred at specific relative phases of the stimulus cycle. The disynaptic correlation is indicated by the strip of yellow, orange, and red pixels running adjacent to the main diagonal $(\Delta t=0)$ (Fig. $10 \mathrm{~B}$, black line) of the matrix, with an offset of $\sim 6 \mathrm{msec}$ (see Materials and Methods). The correlation coefficient (or functional connection strength from the firstorder cross-correlation, as in Fig. 7C) is much higher at a restricted portion of the cortical PSTH (Fig. $10 \mathrm{~B}$, red pixels). Flanking this high-efficacy region are regions of lower efficacy (yellow pixels). We selected spikes corresponding to the narrow $50 \mathrm{msec}$ window of the JPSTH where the efficacy was relatively uniform (within the white dotted lines). Reanalysis of the paired spike enhancement from the "chopped" data is shown in Figure $10 C$. Second spikes are once again more effective than first spikes for intervals up to $\sim 12 \mathrm{msec}$ but thereafter converge to similar levels.
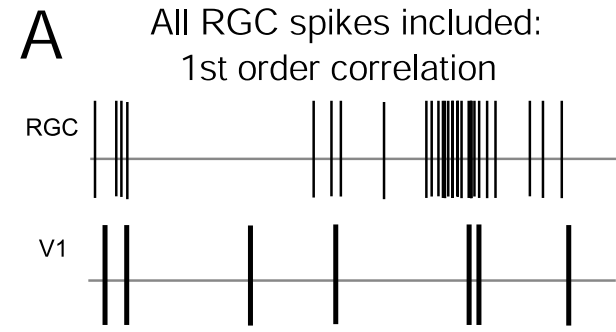

\section{B Paired RGC spikes only: Filtered correlation}
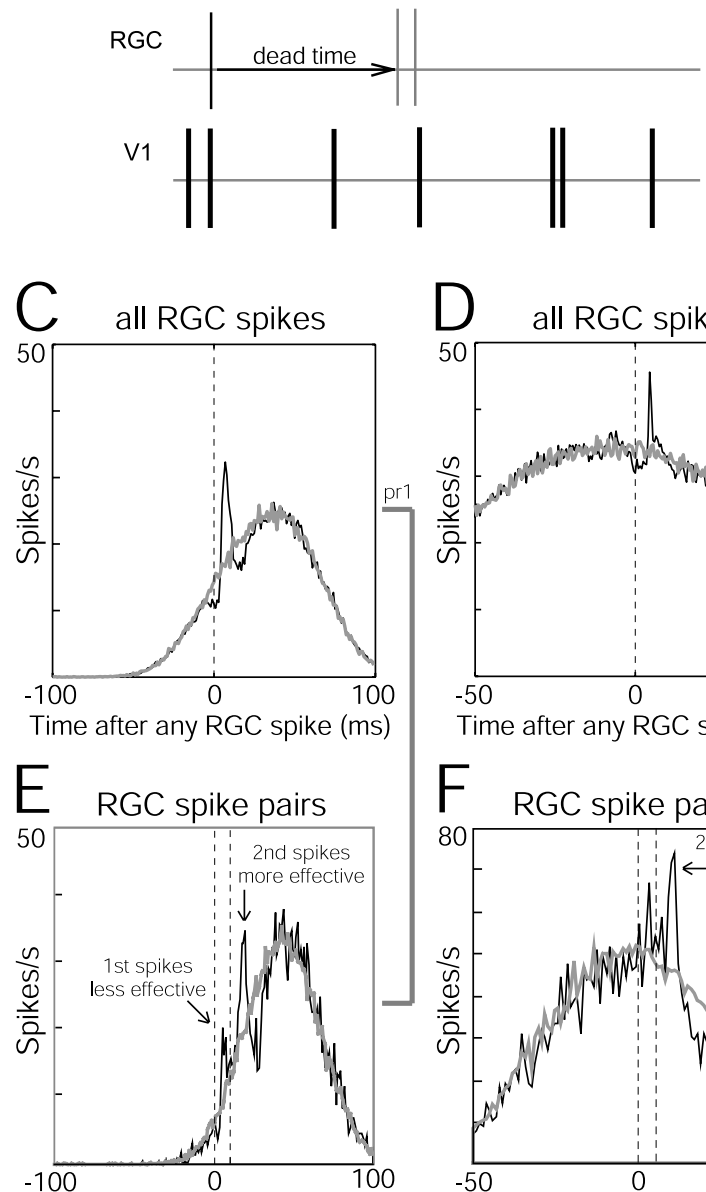

Time after 1st RGC spike (ms)
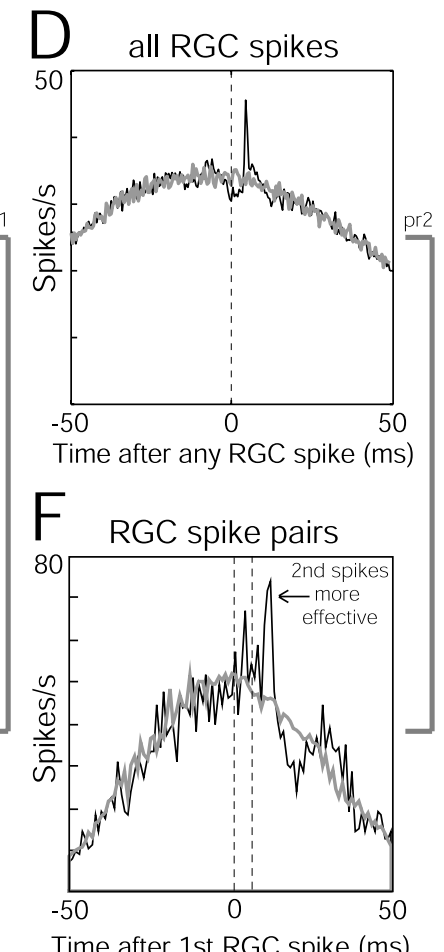

Figure 7. First-order (all retinal spikes) versus filtered (paired retinal spikes) crosscorrelograms. $A, B$, The first-order correlation analysis used all spikes in the retinal spike train, whereas the filtered correlogram used a pair of retinal spikes ( $B$, thin gray lines) with a specific ISI that was preceded by a quiescent period equal to or longer than a specified dead time. $C, D$, First-order cross-correlograms (black traces) for two retinocortical pairs in which time 0 represents the occurrence of all reference (retinal) spikes. $E, F$, Paired spike correlograms (black curves) for fixed retinal interspike intervals ( $10 \mathrm{msec}$ in $E, 5 \mathrm{msec}$ in $F$ ). The first retinal spike in the pair is at time 0 (first dashed line), and the second retinal spike occurs 5 or 10 msec later (second dashed line). Peaks after each retinal spike can be detected in both correlograms. The area under the second peak is greater than twofold larger than the first peak in both cases, suggesting a paired spike enhancement. Gray curves in C-Frepresent shift predictor baselines. The dips below baseline around some peaks are attributable to the refractory period of the RGC. Dead time was 10 msec for both pairs shown in $E$ and $F$.

This control was needed for only one of eight pairs (the other seven of eight pairs showed converging second vs first spike efficacies at 10-20 msec after the first retinal spike). However, the chopping procedure was done for all eight pairs, and the selected 

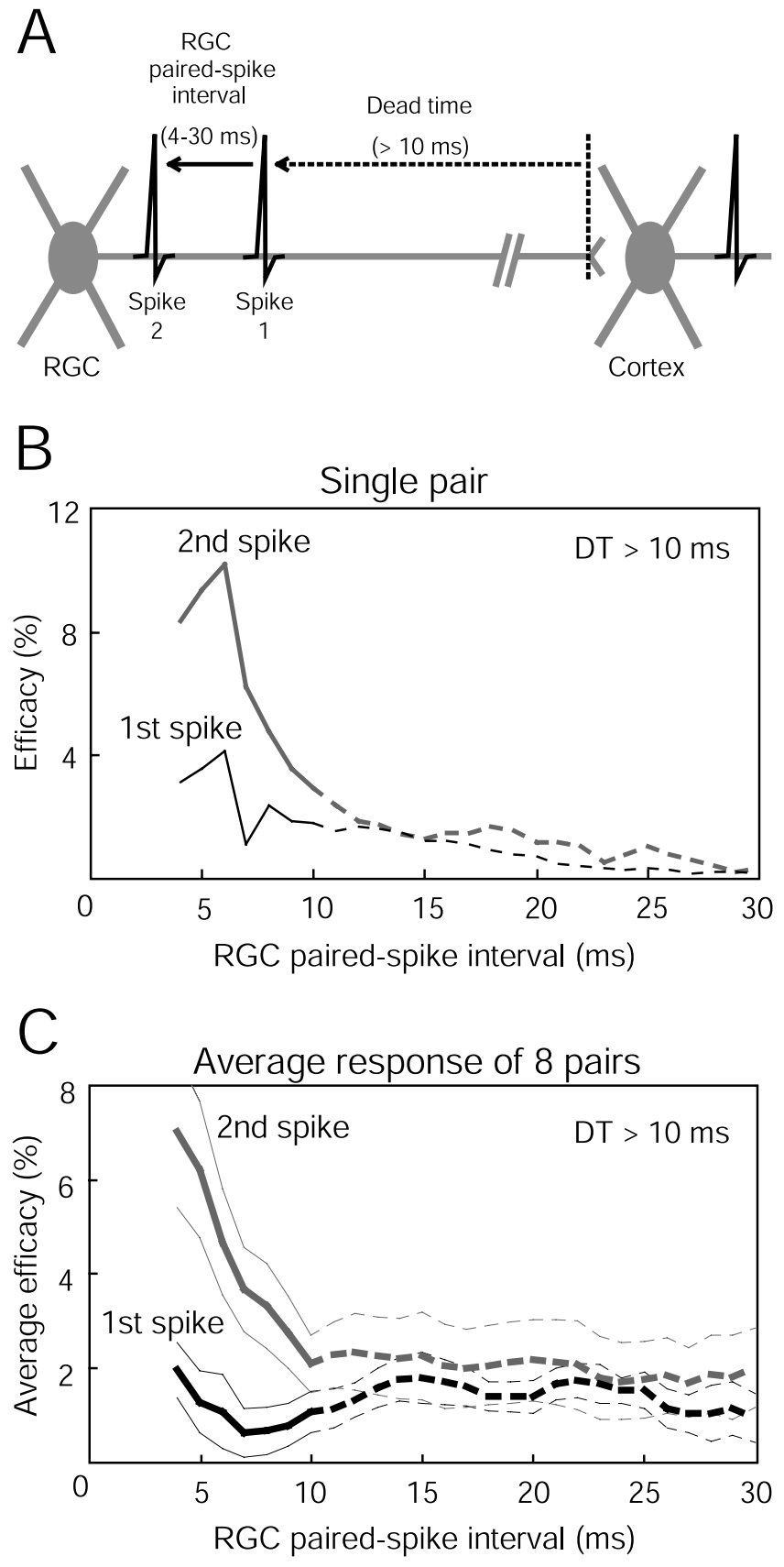

Figure 8. Paired spike enhancement as a function of retinal interspike interval. $A$, Diagram of analysis used to show temporal interactions between two retinal spikes separated by an ISI of between 4 and $30 \mathrm{msec}$, preceded by a dead time of at least $10 \mathrm{msec} . B$, Example of paired spike enhancement for a single retinocortical pair. Data are from the cell pair represented in Figures $2 A$ and $7, D$ and $F$. The second retinal spikes (gray curve) were more effective than the first spikes (black curve) in driving the target cortical cell to fire only at short retinal ISIs. C, Paired spike enhancement averaged over eight retino-cortical pairs in which sufficient spikes were available for analysis. Thick gray and black lines represent mean efficacy, and thin lines represent SEM. Paired spike enhancement was found for retinal intervals of $4-9$ msec. See Results for statistical criteria and significance levels. Dashed lines for ISIs in the range of 11-30 msec correspond to intervals between the two retinal spikes that are greater than the dead time. Data from these intervals (dashed lines) are difficult to interpret because the first spike can be preceded by an interval shorter than the ISI between spikes 1 and 2 (see Materials and Methods).

JPSTH window width depended on the dead time (25 msec window for DT >5, $50 \mathrm{msec}$ for DT $>10$, and $75 \mathrm{msec}$ for DT $>20$ ). Eight to 10 overlapping windows were used for each of the analyzed pairs. In some instances, using the narrow window of
A

PSTH:
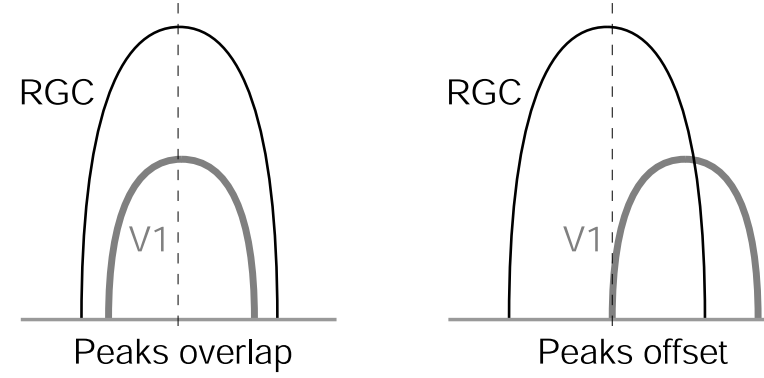

Peaks offset

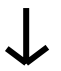

\section{B Paired-spike baseline control:}

Flat baseline: 1st \& 2nd spike equally effective

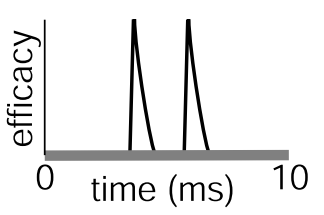

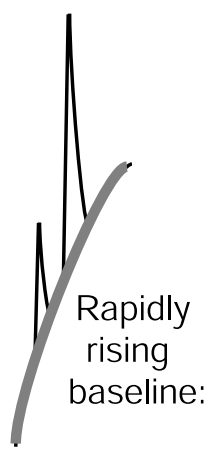

false detect 2 nd spike effic. > 1st.
Figure 9. Schematic diagram of how modulation of connection strength by the visual stimulus might confound paired spike analysis. $A$, Overlapping (left) and nonoverlapping (right) PSTH responses from retinal and cortical cells. $B$, Hypothetical cross-correlogram baselines (thick gray lines) and paired spike efficacies (effic., thin black lines) from PSTH profiles described in $A$. When the correlogram baseline is flat, first and second retinal spikes are equally effective in evoking a spike in the cortex. However, when the baseline is ascending, the second spikes are more effective than the first spikes because the membrane potential of the postsynaptic cortical cell will be closer to the spike threshold. A control for a rising versus a flat correlogram baseline is presented in Figure 10.

JSPTH data resulted in a larger paired spike enhancement (data not shown). Although paired spike enhancement on an ascending cross-correlogram baseline required the above control, paired spike enhancement was robust even on descending baselines (Fig. 7F).

\section{Discussion}

We report that functional connections between retinal ganglion cells and simple cells in layer 4 of the visual cortex are extremely precise. In no cases were connections detected when the overlapping retinal and cortical receptive fields were of opposite signs. The relative position (overlap) of retinal and cortical receptive fields was between 0.6 and 1.0 in $93 \%$ of cases, a slightly higher stringency compared with geniculocortical connections (Alonso et al., 2001). We found that a single retinal afferent contributed to $\sim 3 \%$ of the spiking of cortical neurons. High retinal firing rates improve the likelihood that a retinal spike will have an impact on the firing of cortical cells because significant enhancement of paired spike interactions was detected when retinal spikes were 4-9 msec apart. 
A
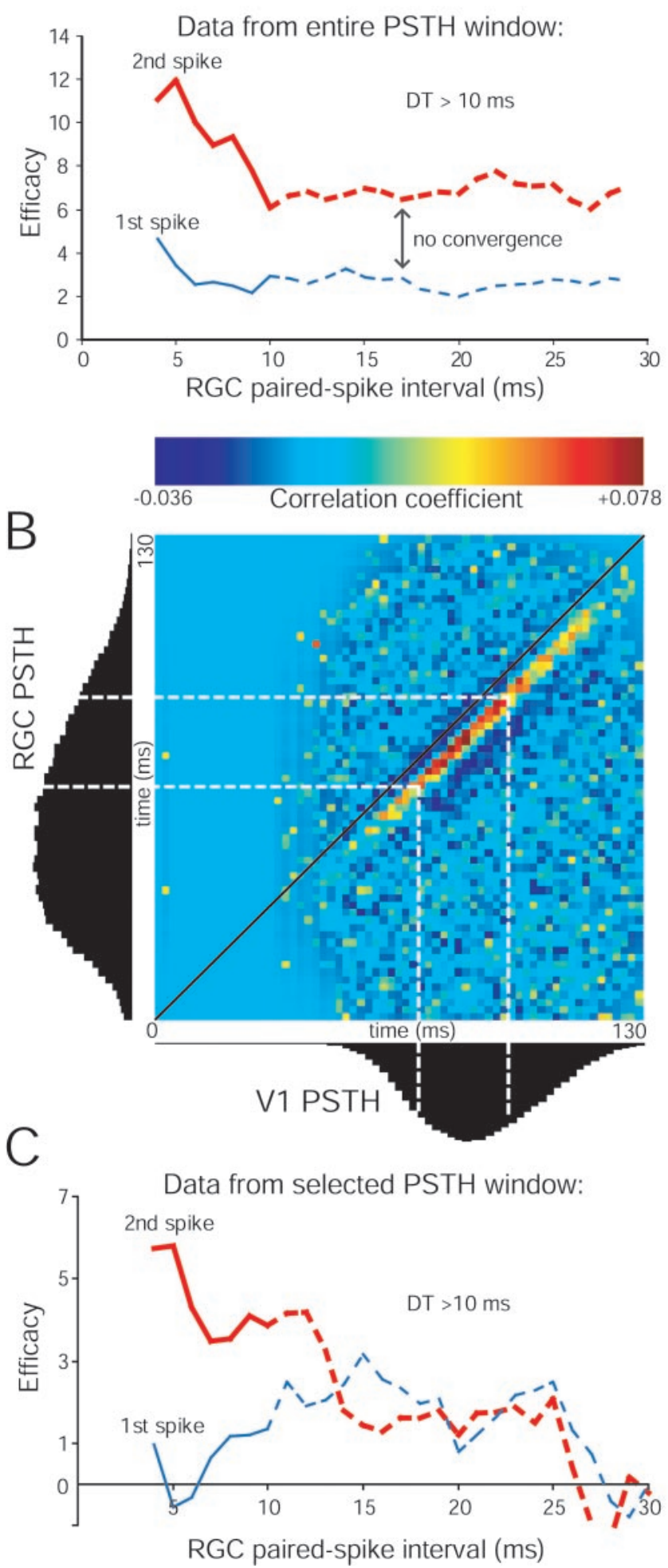

Figure 10. Control for an ascending baseline in paired spike analysis. A, Paired spike enhancement for an RGC-V1 pair that never converges, such that the second retinal spikes are always more effective than the first retinal spikes at all retinal ISIs. B, JPSTH matrix using all spikes from the same retinocortical pair shown as paired spike data in $A$ and first-order crosscorrelation data shown in Figure 7 . The strength of the disynaptic connection is represented by yellow, orange, and red pixels at a 6-9 msec offset from the diagonal (black) line. The efficacy of retinal spikes in driving the cortex is not uniform through the course of the visual responses of the two neurons, as represented by the black PSTHs on the left and below the JPSTH matrix. See Materials and Methods for JPSTH normalization. C, Reanalysis of paired spike interaction using data of uniform efficacy (spikes that occur within the dotted white lines in $B, 50$ msec window).

\section{Detection of disynaptic functional connections}

Although disynaptic analyses of functional connectivity have been explored in motor systems of invertebrate and mammalian preparations (Simmers and Moulins, 1988; Vaughan and Kirkwood, 1997) (for review, see Kirkwood, 1979), to our knowledge, no disynaptic connections have been studied in sensory systems other than in the retinocortical pathway (Lee et al., 1977; present study). The ability to demonstrate disynaptic correlations hinges on several factors. Most importantly, each LGN cell is primarily driven by one or a few retinal inputs, so that the "monosynaptic peaks" in correlograms between retina and LGN are very fast ( $\sim 0.6 \mathrm{msec}$ full width at half-maximum; Usrey et al., 1999) and account for the great majority of LGN spikes (Levick et al., 1972; Cleland and Lee 1985; Mastronarde 1992; Usrey et al., 1999). Furthermore, the connections from LGN to cortex are moderately strong (for EPSP magnitude, see Stratford et al., 1996; Bannister et al., 2002) (for cross-correlation spike contribution from $1-10 \%$, occasionally more, see Alonso et al., 2001), and the crosscorrelograms have a fast rise time, on the order of $1 \mathrm{msec}$ (Tsumoto et al., 1978; Tanaka, 1983; Reid and Alonso, 1995; Alonso et al., 2001). To first approximation, the disynaptic crosscorrelogram should closely resemble the convolution of the two successive monosynaptic correlograms (Reid, 2001), as indeed we found for retinocortical correlations (analysis not shown). In particular, because the retinogeniculate correlogram is extremely fast, disynaptic retinocortical correlograms typically have a rise time ( $<2 \mathrm{msec})$ that is only slightly slower than that of geniculocortical correlograms. In contrast, disynaptic connections cannot be detected further in the hierarchy of visual processing using cross-correlation analysis, for example, from LGN cells to second-order cortical cells (Alonso and Martinez, 1998). This is likely because intracortical cross-correlations are relatively weak and slow compared with retinogeniculate cross-correlations.

It is known that thalamocortical cross-correlations in the somatosensory system (Roy and Alloway, 2001; Swadlow and Gusev, 2001; Bruno and Simons, 2002) and the auditory system (Miller et al., 2001) are quite similar to those found in the geniculocortical system (Tanaka, 1983; Reid and Alonso, 1995). To the extent that inputs to the somatosensory and auditory thalamus resemble retinogeniculate inputs, disynaptic connections to the primary sensory cortex should also be detectable in those systems, as is the case between ascending trigeminal axons from the brainstem and neurons in the somatosensory barrel cortex (H. A. Swadlow, personal communication).

\section{Monosynaptic versus disynaptic rules of connectivity}

The rules of connectivity for disynaptic retinocortical connections (present study) and monosynaptic geniculocortical connections (Alonso et al., 2001) are nearly identical with respect to receptive field sign, position, size, time course, and subregion strength. Considering the strength and specificity of retinogeniculate connections, these results are hardly surprising. However, because of complex intrageniculate properties such as fast synchrony (Alonso et al., 1996) and bursting (Guido et al., 1992; Guido and Lu, 1995) together with retinogeniculate divergence and geniculocorti-

Using this limited data set with fewer than one-third of the spikes used in $A$, the overall first and second spike efficacies were expectedly smaller. At short retinal intervals, second spikes are still more effective than first spikes. At longer intervals, first and second spike efficacies were of virtually identical magnitudes. For a few ISIs, efficacies dipped below zero because of subtraction of the shift predictor from a noisy correlogram derived from a small number of paired spikes. 


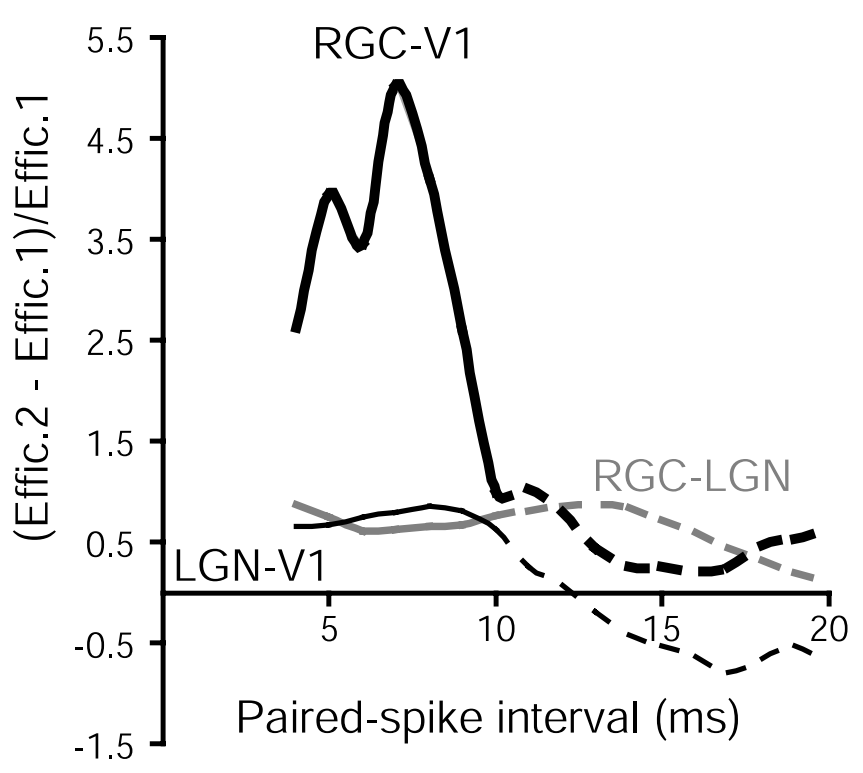

Figure 11. Mean paired-spike enhancement effects for monosynaptic and disynaptic pathways along the cat primary visual pathway using $4 \mathrm{~Hz}$ drifting grating visual stimuli of $50 \%$ contrast. Dead time was $>10 \mathrm{msec}$ for all data sets. Across the RGC $\rightarrow$ LGN synapse, paired spike enhancement persists for $15-20 \mathrm{msec}$ [thin gray curve; data reanalyzed from Usrey et al. (1998); $n=12$ pairs]. For the monosynaptic LGN $\rightarrow$ V1 synapse, paired spike enhancement appears more short-lived [thin black curve; data replotted from Usrey etal. (2000); $n=11$ pairs]. For the disynaptic RGC-V1 pathway (thick black curve; $n=8$ pairs), paired spike enhancement is relatively much larger than for RGC-LGN and LGN-V1 monosynaptic pairs. Dashed lines for ISIs in the range 11-20 msec correspond to paired spike intervals that are greater than the dead time, which are difficult to interpret (see Materials and Methods). Effic., Efficacy.

cal convergence (Fig. 1), the rules of disynaptic retinocortical connections required validation before more complex temporal interactions such as paired spike enhancement could be explored.

\section{Monosynaptic versus disynaptic paired spike enhancement}

Paired spike enhancement was previously described for retinogeniculate (Mastronarde, 1987; Usrey et al., 1998; Levine and Cleland, 2001; Rowe and Fischer, 2001) and geniculocortical (Usrey et al., 2000) connections. The simplest biophysical mechanism driving paired spike enhancement is temporal summation in postsynaptic neurons (Usrey et al., 1998, 2000; Weyand, 2001), which likely occurs after the saturation of rapid sensory adaptation and synaptic depression. Thus paired spike enhancement may occur in a state of tonic synaptic depression of both the retinogeniculate synapses (Turner and Salt, 1998; Chen et al., 2002) and geniculocortical synapses (Stratford et al., 1996; Bannister et al., 2002) (for somatosensory thalamocortical depression, see Chung et al., 2002). In our current disynaptic study, we did not explore the onset of depression or adaptation, primarily because neurons were never silent for more than $\sim 150 \mathrm{msec}$ with our $4 \mathrm{~Hz}$ repetitive stimulus. Instead, we focused on the short-term enhancement of signal transfer in functionally connected RGCs and cortical simple cells during steady-state visual stimulation.

To compare the strength of the effect and its time course for different pathways, it is important to ensure that the stimulus and analysis techniques are as similar as possible. Two past studies of monosynaptic connections from our laboratory have used identical stimuli (50\% contrast drifting gratings) and recording conditions similar to those we have used here to study the disynaptic connection from retina to cortex. In Figure 11, we compare the strength and time course of paired spike enhancement from all three studies for a dead time of $10 \mathrm{msec}$.
The magnitude of disynaptic paired spike enhancement (greater than fourfold under these conditions) was much larger than seen across retinogeniculate and geniculocortical synapses ( 1.5-fold) (Fig. 11). A possible mechanism for this stronger effect might be found in the divergence from retina to LGN, followed by reconvergence onto cortical neurons. Retinogeniculate paired spike enhancement tends to create increased synchrony in the LGN (Usrey et al., 1998), whereas synchronous input to a cortical neuron is synergistic in driving cortical neurons (Alonso et al., 1996). However, the magnitude of the observed disynaptic paired spike enhancement we observed was greater than might be explained by a very simple model that incorporates divergence and reconvergence. Assuming divergence of one RGC onto two identical relay neurons and reconvergence back onto a cortical target, one can calculate retinocortical paired spike enhancement by computing the probability of each pattern of one or two spikes in the LGN cells, followed by the conditional probability of cortical spikes following each pattern in the LGN. If retinogeniculate transmission has a baseline efficacy of 30\%, and paired-spike enhancement increases efficacy by a factor of 1.5 at both levels (Fig. 11), including heterosynaptic interactions from LGN to cortex (Usrey et al., 2000), the predicted retinocortical paired spike enhancement would be $\sim 2.3$ (analysis not shown).

In our paired spike analysis, we tried to analyze how the efficacy of transmission was modulated by the immediate history of the presynaptic spike train, all things being equal. This is why we were careful to enforce a dead time before the first spike in a pair and also to ensure that both spikes occurred at an approximately equivalent presynaptic and postsynaptic baseline rate (Fig. 10). It is therefore important to emphasize that other factors influence the efficacy of synaptic connections, such as the instantaneous response rate of both neurons, as has been demonstrated by others using JPSTH analysis (Aertsen et al., 1989). In the present study, we chose to analyze paired spike enhancement, primarily so that our results could be compared with previous monosynaptic studies (Alonso et al., 1996; Usrey et al., 1998, 2000). Nonetheless, the enhancement of efficacy is almost certainly more closely related to the number of presynaptic spikes fired within some integration period, rather than the immediately preceding spike (Usrey et al., 1998; Rowe and Fischer, 2001).

\section{Functional significance}

One possible consequence of paired spike enhancement is that closely spaced action potentials in presynaptic neurons might be preferentially evoked by certain stimuli, such as high-contrast bars, but not by others stimuli, such as white noise. This hypothesis would be difficult to prove, however, because other factors known to influence monosynaptic connections, such as the postsynaptic firing rate, change for different stimuli. Alternatively, different firing states, such as bursting in the LGN (Guido et al., 1992), might be preferentially transmitted to the cortex. To test such a hypothesis, however, it would be necessary to dissociate bursting in the LGN from other changes in thalamic and cortical activity.

Although it might be difficult to assess whether paired spike enhancement increases the transmission of specific types of information to the cortex, it is generally true that higher retinal rates are preferentially transmitted to the cortex, other things being equal. This would ensure that when the retina is sending its most reliable signals (Berry and Meister, 1998; Kara et al., 2000), the cortex is most likely to respond to these signals. Divergence of these signals to multiple targets in the LGN, followed by recon- 
vergence onto common cortical targets (Alonso et al., 1996; Usrey et al., 2000; Reid, 2001), is an additional mechanism by which these strong signals are preferentially amplified. One might imagine that such mechanisms would not be necessary if all retinal spikes could be transmitted faithfully through the LGN and on to the cortex, but there are independent reasons for having the thalamus serve as a partial, or leaky, relay of information from the periphery. Most importantly, a moderate efficacy of transmission allows the thalamus to modulate the gain of cortical input, such as during the sleep-wake cycle (Steriade and Llinas, 1988; McCormick and Bal, 1994) and selective visual attention (Crick, 1984; Guillery and Sherman, 2002). Given this constraint, retinogeniculate divergence and geniculocortical convergence are means to increase the fidelity of transmission but without compromising cortical stimulus selectivity.

\section{References}

Aertsen AM, Gerstein GL, Habib MK, Palm G (1989) Dynamics of neuronal firing correlation: modulation of "effective connectivity." J Neurophysiol 61:900-917.

Alonso JM, Martinez LM (1998) Functional connectivity between simple cells and complex cells in cat striate cortex. Nat Neurosci 1:395-403.

Alonso JM, Usrey WM, Reid RC (1996) Precisely correlated firing in cells of the lateral geniculate nucleus. Nature 383:815-819.

Alonso JM, Usrey WM, Reid RC (2001) Rules of connectivity between geniculate cells and simple cells in cat primary visual cortex. J Neurosci 21:4002-4015.

Bannister NJ, Nelson JC, Jack JJ (2002) Excitatory inputs to spiny cells in layers 4 and 6 of cat striate cortex. Philos Trans R Soc Lond B Biol Sci 357:1793-1808.

Berry II MJ, Meister M (1998) Refractoriness and neural precision. J Neurosci 18:2200-2211.

Blalock HM (1972) Social statistics. New York: McGraw-Hill.

Bruno RM, Simons DJ (2002) Feedforward mechanisms of excitatory and inhibitory cortical receptive fields. J Neurosci 22:10966-10975.

Chen C, Blitz DM, Regehr WG (2002) Contributions of receptor desensitization and saturation to plasticity at the retinogeniculate synapse. Neuron 33:779-788.

Chung S, Li X, Nelson SB (2002) Short-term depression at thalamocortical synapses contributes to rapid adaptation of cortical sensory responses in vivo. Neuron 34:437-446.

Cleland BG, Lee BB (1985) A comparison of visual responses of cat lateral geniculate nucleus neurones with those of ganglion cells afferent to them. J Physiol (Lond) 369:249-268.

Crick F (1984) Function of the thalamic reticular complex: the searchlight hypothesis. Proc Natl Acad Sci USA 81:4586-4590.

Epping WJ, Eggermont JJ (1987) Coherent neural activity in the auditory midbrain of the grassfrog. J Neurophysiol 57:1464-1483.

Guido W, Lu SM (1995) Cellular bases for the control of retinogeniculate signal transmission. Int J Neurosci 80:41-63.

Guido W, Lu SM, Sherman SM (1992) Relative contributions of burst and tonic responses to the receptive field properties of lateral geniculate neurons in the cat. J Neurophysiol 68:2199-2211.

Guillery RW, Sherman SM (2002) Thalamic relay functions and their role in corticocortical communication: generalizations from the visual system. Neuron 33:163-175.

Hochstein S, Shapley RM (1976) Quantitative analysis of retinal ganglion cell classifications. J Physiol (Lond) 262:237-264.

Kara P, Reinagel P, Reid RC (2000) Low response variability in simultaneously recorded retinal, thalamic, and cortical neurons. Neuron 27:635-646.

Kara P, Pezaris JS, Yurgenson S, Reid RC (2002) The spatial receptive field of thalamic inputs to single cortical simple cells revealed by the interaction of visual and electrical stimulation. Proc Natl Acad Sci USA 99:16261-16266.

Kirkwood PA (1979) On the use and interpretation of cross-correlations measurements in the mammalian central nervous system. J Neurosci Methods 1:107-132.

Lee BB, Cleland BG, Creutzfeldt OD (1977) The retinal input to cells in area 17 of the cat's cortex. Exp Brain Res 30:527-538.
Levick WR, Cleland BG, Dubin MW (1972) Lateral geniculate neurons of cat: retinal inputs and physiology. Invest Ophthalmol 5:302-311.

Levine MW (1998) Cross-correlation between neurons: a source of information about the nervous system. Biosystems 48:139-146.

Levine MW, Cleland BG (2001) An analysis of the effect of retinal ganglion cell impulses upon the firing probability of neurons in the dorsal lateral geniculate nucleus of the cat. Brain Res 902:244-254.

Lu SM, Guido W, Sherman SM (1992) Effects of membrane voltage on receptive field properties of lateral geniculate neurons in the cat: contributions of the low-threshold $\mathrm{Ca} 2+$ conductance. J Neurophysiol 68:2185-2198.

Mastronarde DN (1987) Two classes of single-input X-cells in cat lateral geniculate nucleus. II. Retinal inputs and the generation of receptive-field properties. J Neurophysiol 57:381-413.

Mastronarde DN (1992) Nonlagged relay cells and interneurons in the cat lateral geniculate nucleus: receptive-field properties and retinal inputs. Vis Neurosci 8:407-441.

McCormick DA, Bal T (1994) Sensory gating mechanisms of the thalamus. Curr Opin Neurobiol 4:550-556.

Merrill EG, Ainsworth A (1972) Glass-coated platinum-plated tungsten microelectrodes. Med Biol Eng 10:662-672.

Miller LM, Escabi MA, Read HL, Schreiner CE (2001) Functional convergence of response properties in the auditory thalamocortical system. Neuron 32:151-160.

Perkel DH, Gerstein GL, Moore GP (1967) Neuronal spike trains and stochastic point processes. II. Simultaneous spike trains. Biophys J 7:419-440.

Reid RC (2001) Divergence and reconvergence: multielectrode analysis of feedforward connections in the visual system. Prog Brain Res 130:141-154.

Reid RC, Alonso JM (1995) Specificity of monosynaptic connections from thalamus to visual cortex. Nature 378:281-284.

Reid RC, Victor JD, Shapley RM (1997) The use of m-sequences in the analysis of visual neurons: linear receptive field properties. Vis Neurosci 14:1015-1027.

Rowe MH, Fischer Q (2001) Dynamic properties of retino-geniculate synapses in the cat. Vis Neurosci 18:219-231.

Roy SA, Alloway KD (2001) Coincidence detection or temporal integration? What the neurons in somatosensory cortex are doing. J Neurosci 21:2462-2473.

Saul AB, Humphrey AL (1992) Evidence of input from lagged cells in the lateral geniculate nucleus to simple cells in cortical area 17 of the cat. J Neurophysiol 68:1190-1208.

Simmers J, Moulins M (1988) A disynaptic sensorimotor pathway in the lobster stomatogastric system. J Neurophysiol 59:740-756.

Steriade M, Llinas RR (1988) The functional states of the thalamus and the associated neuronal interplay. Physiol Rev 68:649-742.

Stratford KJ, Tarczy-Hornoch K, Martin KA, Bannister NJ, Jack JJ (1996) Excitatory synaptic inputs to spiny stellate cells in cat visual cortex. Nature 382:258-261

Swadlow HA, Gusev AG (2001) The impact of "bursting" thalamic impulses at a neocortical synapse. Nat Neurosci 4:402-408.

Tanaka K (1983) Cross-correlation analysis of geniculostriate neuronal relationships in cats. J Neurophysiol 49:1303-1318.

Tsumoto T, Creutzfeldt OD, Legendy CR (1978) Functional organization of the corticofugal system from visual cortex to lateral geniculate nucleus in the cat (with an appendix on geniculo-cortical mono-synaptic connections). Exp Brain Res 32:345-364.

Turner JP, Salt TE (1998) Characterization of sensory and corticothalamic excitatory inputs to rat thalamocortical neurons in vitro. J Physiol (Lond) 510:829-843.

Usrey WM, Reppas JB, Reid RC (1998) Paired-spike interactions and synaptic efficacy of retinal inputs to the thalamus. Nature 395:384-387.

Usrey WM, Reppas JB, Reid RC (1999) Specificity and strength of retinogeniculate connections. J Neurophysiol 82:3527-3540.

Usrey WM, Alonso JM, Reid RC (2000) Synaptic interactions between thalamic inputs to simple cells in cat visual cortex. J Neurosci 20:5461-5467.

Vaughan CW, Kirkwood PA (1997) Evidence from motoneurone synchronization for disynaptic pathways in the control of inspiratory motoneurones in the cat. J Physiol (Lond) 503:673-689.

Weyand TG (2001) Temporal summation at the awake retino-geniculate synapse. Soc Neurosci Abstr 27:723.1. 